\title{
Broadband Uniplanar Microstrip to Slot-Line Transitions
}

Nihad I. Dib

University of Michigan

Ann Arbor, Michigan

Rainee N. Simons

NYMA, Inc.

Brook Park, Ohio

Linda P.B. Katehi

University of Michigan

Ann Arbor, Michigan

March 1995

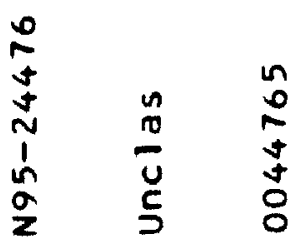

$\sum_{0}^{m}$

Prepared for

Lewis Research Center

Under Contract NAS3-27186
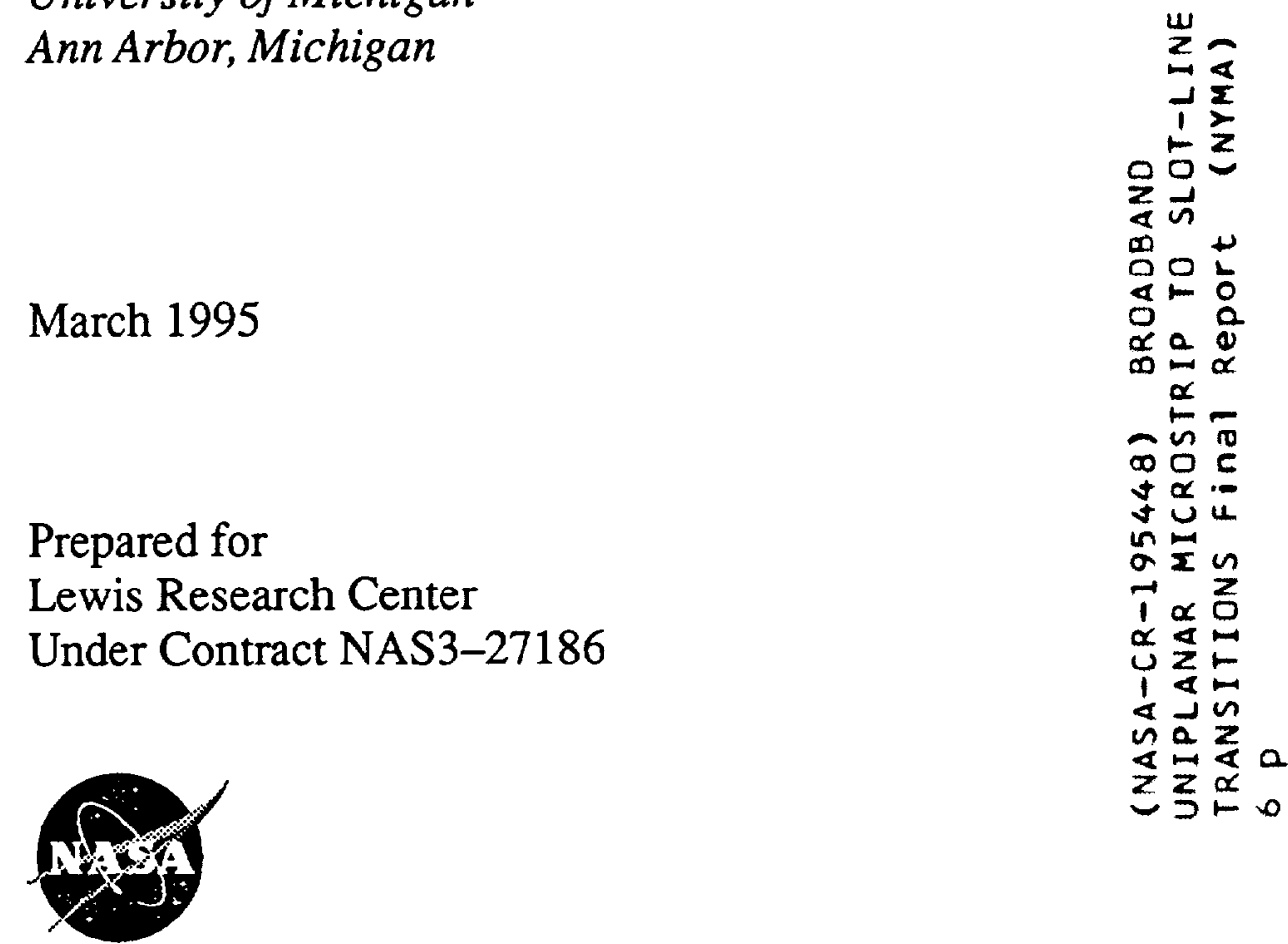

National Aeronautics and

Space Administration 


\title{
Broadband Uniplanar Microstrip to Slot-Line Transitions
}

\author{
Nihad I. Dib \\ University of Michigan \\ Radiation Laboratory \\ Ann Arbor, Michigan 48109 \\ Rainee N. Simons \\ NYMA, Inc. \\ Brook Park, Ohio 44142 \\ Linda P.B. Katehi \\ University of Michigan \\ Radiation Laboratory \\ Ann Arbor, Michigan 48109
}

\begin{abstract}
New in line uniplanar microstrip-to-slotline transitions for MIC/MMIC and phased array slotline antenna applications are described. Such transitions are compact and suitable to be used in an open environment or inside a package or a multichip module. The transitions share the concept of using a balun which consists of two microstrip lines connected to a slotline through a pair of coupled microstrips. In this paper, the transitions are studied theoretically using the FDTD technique and measured experimentally using an HP8510C Network Analyzer. For a back-to-back configuration, an insertion loss of less than $1.3 \mathrm{~dB}$ per transition is achieved over a $40 \% 3-\mathrm{dB}$ bandwidth with a minimum of $0.6 \mathrm{~dB}$ at the design frequency.
\end{abstract}

\section{Introduction}

In most slotline antenna applications, a transition is needed to couple the slotline to another planar line, e.g., microstrip line. Although there are techniques to couple the RF energy from a microstrip line to a slot-line, both sides of the substrate are needed to achieve a successful transition. Such a configuration requires accurate alignment during fabrication due to the demonstrated sensitivity of the transition on the relative position between the microstrip, which is printed on one side of the substrate, to the slotline printed on the other side. Furthermore, such a transition requires a multi-wafer/substrate configuration and presents many practical problems when there is a need for packaging.

In 1983, a single sided microstrip to slot-line transition was proposed and measured $[1,2]$. This transition uses a balun in the form of an open ring consisting of two microstrip lines connected to a slot-line through a pair of coupled microstrip. Recently, the same transition has been studied by the authors both theoretically and experimentally $[3,4]$. It has been found that the insertion loss and return loss for a single transition are better than $1.5 \mathrm{~dB}$ and $10 \mathrm{~dB}$, respectively, over $8 \%$ bandwidth centered at $9 \mathrm{GHz}$ (the design frequency was $10 \mathrm{GHz}$ ) [4]. Moreover, a linearly tapered slot antenna (LTSA) excited by this microstripto-slotline transition has been investigated [4]. A major limitation in this approach when used in slotline antennas is the off-axis feeding mechanism and the resulting large space requirement for such a feed.

In addition, a new approach for designing a uniplanar microstrip-to-slotline transition for MIC/MMIC and antenna applications has been proposed in [5]. In this transition, a slot-line radial stub is utilized to create resonance conditions at the junction of the two transmission lines so that maximum power transfer is achieved. It was reported that the bandwidth of the tested transition was $30 \%$ at a design frequency of $3.8 \mathrm{GHz}[5]$.

In this paper, new microstrip-to-slotline uniplanar topologies are proposed which provide broadband transition characteristics as the ones presented in the literature but require much less real estate and give an on-axis input/output geometry. Because of these properties, these new transitions can easily be used as feeds for slotline antennas in phased array applications.

\section{Design Description}

Figure 1(a) shows a back-to-back uniplanar microstrip to slot-line transition geometry. It consists of a $50 \Omega \mathrm{mi}$ crostrip line which branches into two orthogonal paths. The characteristic impedance of each microstrip path is chosen as $70 \Omega$ for easy fabrication. The two microstrip paths are made of $0.75 \lambda_{g \text { (mierostrip) }}$ and $0.25 \lambda_{g \text { (microstrip) }}$ long, respectively, at the design frequency of $10 \mathrm{GHz}$ so that the fields at the locations $a$ and $b$ are $180^{\circ}$ ont of phase. This is necessary to excite the odd mode on the coupled microstrip lines so that the intermediate coupled microstrip line section transitions easily into a slot-line as shown in the figure. In practice however, discontinuity parasitics contribute an additional phase change which may be adjusted by slightly changing the lengths of the microstrip lines. The configuration shown in Fig. 1(a) has the two transitions on the same side of the slotline and is called an unbalanced configuration in contrast to the balanced configuration shown in Figure 1(b) where the two transitions are located on opposite sides of the slotline. In the latter configuration, the two microstrip path lengths are made $\mathbf{0 . 8}$ $\lambda_{\text {(microstrip) }}$ and $0.25 \lambda_{g \text { (microstrip) long, respectively, at }}$ the design frequency of $10 \mathrm{GHz}$ to compensate for the right angle bends parasitics. The rest of the design is the same as above.

Figures $1(c)$ and $1(d)$ show two compact microstrip to slot-line transition topologies. In these transitions, the $\mathbf{0 . 2 5}$ $\lambda_{\text {(microstrip) }}$ line is eliminated and the delay path is made around $0.6 \lambda_{g(\text { mierostrip) }}$ at the design frequency of $10 \mathrm{GHz}$ 


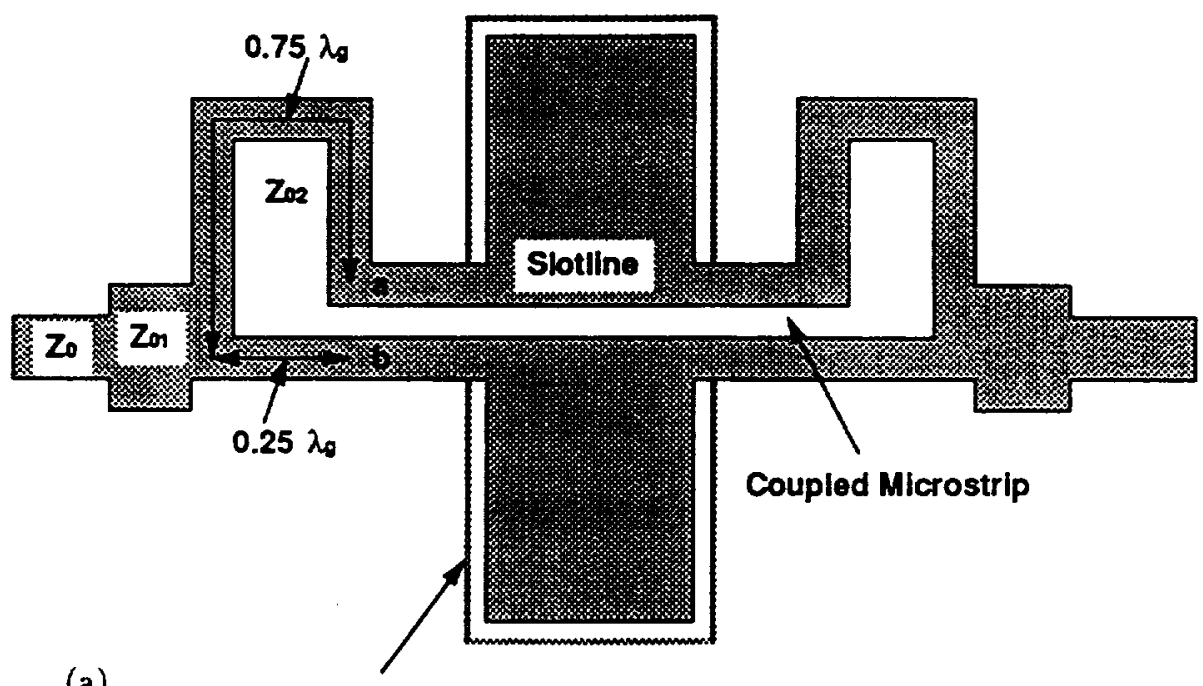

Etched slot in the ground plane on the other side of the substrate

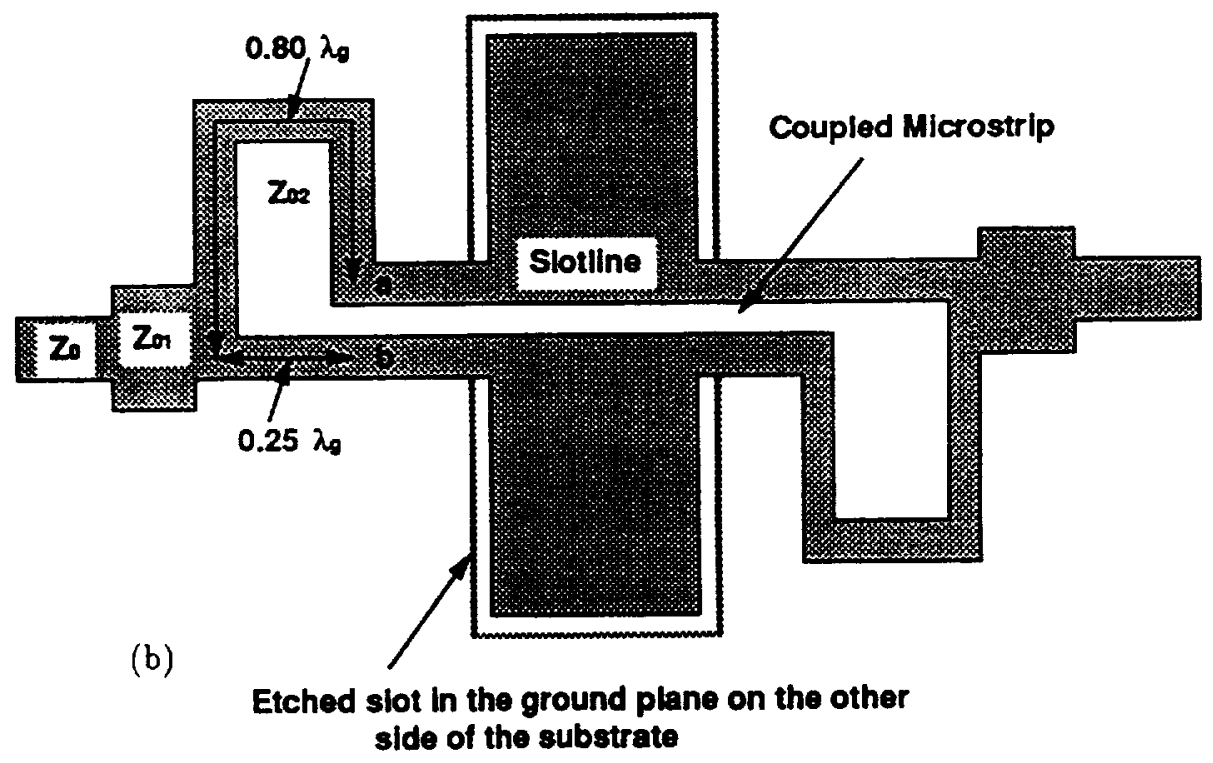

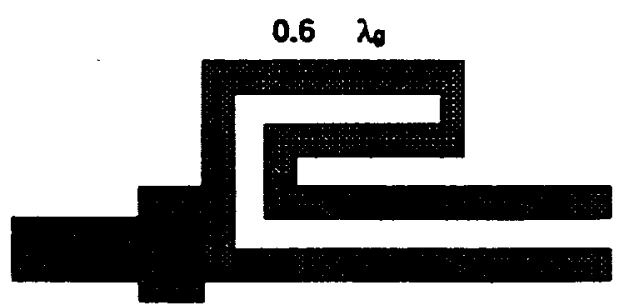

(c)

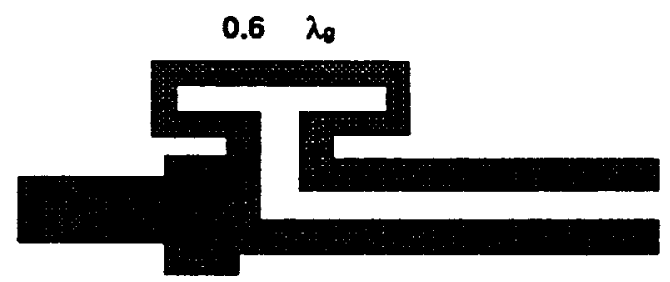

(d)

Figure 1: Microstrip-to-slotline transitions (a) back-to-back unbalanced configuration (b) back-to-back compensated and balanced configuration (c), (d) compact transitions. $Z_{0}=50 \Omega, Z_{01}=41.8 \Omega, Z_{02}=70 \Omega, \lambda_{g}=11.6 \mathrm{~mm}$ at $10 \mathrm{GHz}$ 
to provide the $180^{\circ}$ phase difference and to compensate for the right angle bends parasitics. The rest of the design is the same as above. These compact transitions offer as much as $45 \%$ reduction in size over the one shown in Fig. 1(a).

Impedance matching at the junction between the $50 \Omega$ microstrip line and the two $70 \Omega$ microstrip lines is achieved by a quarter wave impedance transformer of characteristic impedance $41.8 \Omega$. The characteristic impedances of the various lines are also indicated in Fig. 1. The transitions are fabricated on a $1.5 \times 2.0$ inch RT-Duriod6010.5 of 10 mils thickness and $\epsilon_{r}$ of 10.5 .

\section{FDTD Method}

In this method, Maxwell's curl equations are expressed in discretized space and time domains and are then used to simulate the propagation of an initial excitation in a "leapfrog" manner [6]. Recently, the method has been successfully applied to characterize microstrip and coplanar waveguide (CPW) lines and discontinuities $[7,8,9]$. In order to characterize any planar discontinuity, propagation of a specific time-dependent function through the structure is simulated using the FDTD technique. A Gaussian pulse is used here because it is smoothly varying in time and its Fourier transform is also a Gaussian function centered at zero frequency. Following the time and space discretizations of the electric and magnetic field components, the FDTD equivalents of Maxwell's equations are then used to update the spatial distributions of these components at alternating half time steps [6]. The space steps, $\Delta x, \Delta y$ and $\Delta z$, are carefully chosen such that integral numbers of them can approximate the various dimensions of the structure. As a rule of thumb and in order to reduce the truncation and grid dispersion errors, the maximum step size is chosen to be less than $1 / 20$ of the smallest wavelength existing in the computational domain (i.e., at the highest frequency represented in the pulse). Then, the Courant stability criterion is used to select the time step to insure numerical stability.

The super-absorbing first-order Mur boundary condition is utilized to terminate the FDTD lattice at the front and back planes in order to simulate infinite lines. On the other hand, the first-order Mur boundary condition is used on the other walls to simulate an open structure.

In general, the frequency dependent scattering parameters, $S_{i j}$, can be obtained from the voltages at ports $i$ and $j$. To obtain $\mathrm{S}_{11}(\omega)$, the incident and reflected fields must be known. The incident field is obtained from a line of an infinite extent and is subtracted from the total waveform to yield the reflected field.

\section{Results}

The measurements were done using an HP8510C Network Analyzer with the Wiltron Universal test fixture. Figure

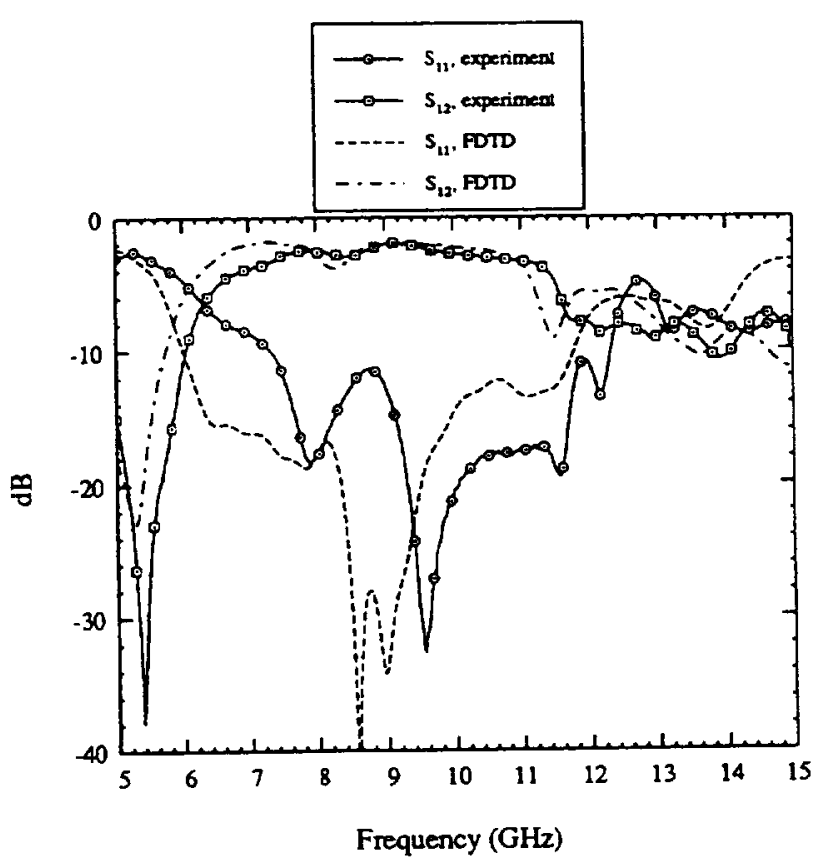

Figure 2: S-parameters of an unbalanced and compensated back-to-back transition.

2 shows the S-parameters of an unbalanced and compensated back-to-back configuration which is the same as the one shown in Fig. 1(a) except that the length of one of the microstrip paths is $0.8 \lambda_{g}$ instead of $0.75 \lambda_{g}$. The overall agreement between the experimental and theoretical results is satisfactory considering the differences between the theoretically analyzed structure and the experimentally characterized one. Specifically, the FDTD method considered a transition printed on an infinite substrate while the experimentally measured transition was printed on a finite substrate. Furthermore, the FDTD method implemented a slightly different transition geometry due to limitations of the uniform grid in discretizing arbitrary dimensions without introducing an impossibly large number of nodes. The measurement data are calibrated to the connectors and include the connector insertion loss which amounts to $0.2 \mathrm{~dB}$ per connector. The measured insertion loss of the backto-back configuration is better than $3 \mathrm{~dB}$ in the frequency range $7.5-10.5 \mathrm{GHz}$ and translates into $1.3 \mathrm{~dB}$ per transition over a $30 \%$ bandwidth.

Figure 3 shows the S-parameters of the balanced and compensated back-to-back configuration shown in Fig. 1(b). The measured insertion loss is better than $3 \mathrm{~dB}$, that is $1.3 \mathrm{~dB}$ per transition, from $7 \mathrm{GHz}$ to $11.5 \mathrm{GHz}(40 \%$ bandwidth). The minimum insertion loss achieved by a single transition is $0.6 \mathrm{~dB}$ at $10.5 \mathrm{GHz}$.

It should be mentioned that while experimentally characterizing the transitions, a piece of microwave absorber was placed at the edges of the substrate to absorb the surface waves launched by the microstrip line discontinuities. 


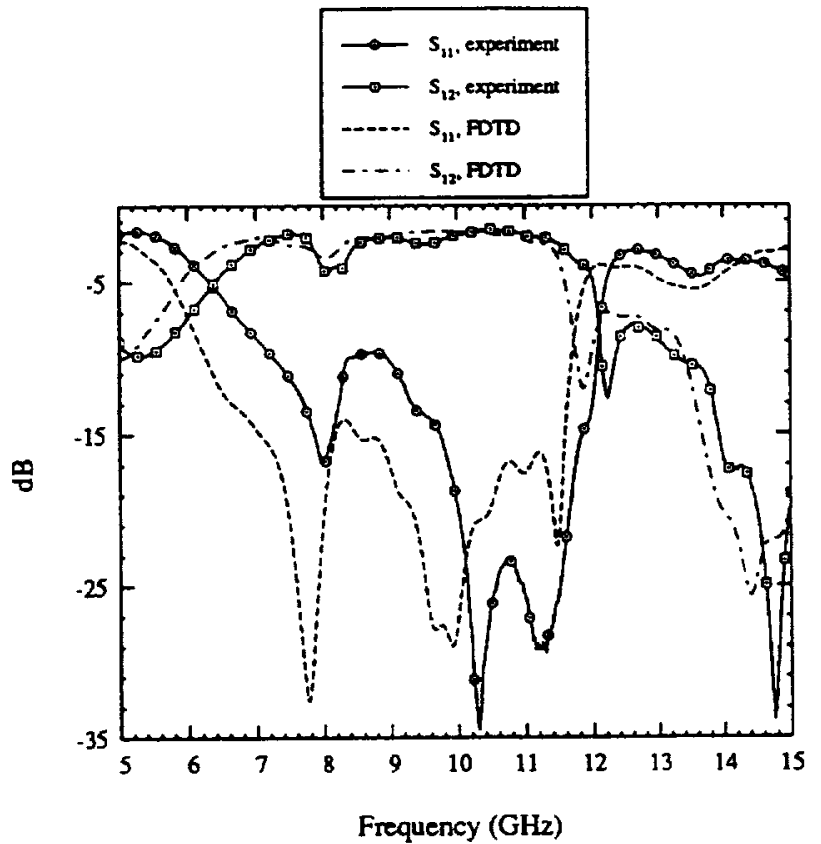

Figure 3: S-parameters of a balanced and compensated back-to-back configuration.

Without the absorber, deep nulls in the insertion loss measurements were observed. Also, as mentioned earlier, the measured data include the losses from the coaxial connectors of the Wiltron Universal test fixture which were not practical to calibrate out.

Results for the compact transitions shown in Figs. 1(c) and (d) will be presented in the symposium.

\section{Conclusions}

Uniplanar microstrip-to-slotline transitions have been proposed and characterized both experimentally and theoretically. Such transitions are compact and easily adaptable to be used in MIC/MMIC and antenna applications. The response of the transitions is evaluated numerically and ex-: perimentally from $5 \mathrm{GHz}$ to $15 \mathrm{GHz}$ and both results are in good agreement. For a balanced and compensated back-toback configuration, an insertion loss better than $1.3 \mathrm{~dB}$ per transition is achieved over a $40 \% 3-\mathrm{dB}$ bandwidth around the design frequency $(10 \mathrm{GHz})$.

\section{Acknowledgements}

This work was supported by the Army Research Office.

\section{References}

[1] N. El-Minyawi, "A New Microstrip-Slotline Transi- tion, "Microwave Journal, Vol. 26, No. 10, pp. 140141, Oct. 1983.

[2] N. El-Minyawi, "Single Sided Slotline Microstrip Transition," Technical Memorandum, IEE Proc., Pt. H, Vol. 134, No. 1, pp. 101-102, Feb. 1987

[3] J. Yook, N. Dib, L. Katehi, R. Simons and S. Taub, "Theoretical and Experimental Study of Microstripto-Slot Line Uniplanar Transition, " 1994 IEEE AP-S International Symposium Digest, pp. 1206-1209.

[4] R. Simons, S. taub, R. Lee and P. Young, "Microwave Characterization of Slot Line and Coplanar Strip Line on High-Resistivity Silicon For a Slot Antenna Feed Network," Microwave and Optical Technology Letters, Vol. 7, No. 11, pp. 489-494, Aug. 1994.

[5] T. Ho and S. Hart, "A Novel Uniplanar Microstrip to Slot-Line Transition," Microwave and Optical Technology Letters, Vol. 7, No. 13, pp. 619-620, Sep. 1994.

[6] K. Kunz and R. Luebbers, The Finite Difference Time Domain Method for Electromagnetics, Florida: CRC press, 1993.

[7] X. Zhang and K. Mei, "Time-domain finite difference approach to the calculation of the frequencydependent characteristics of microstrip discontinuities, "IEEE Trans. Microwave Theory and Techniques, pp. 1775-1787, Dec. 1988.

[8] D. Sheen, S. Ali, M. Abouzahra and J. Kong, "Application of the Three-Dimensional Finite-Difference Time-Domain Method to the Analysis of Planar Microstrip Circuits, "IEEE Trans. Microwave Theory and Techniques, pp. 849-857, July 1990.

[9] S. Visan, O. Picon and V. Hanna, “3D Characterization of Air Bridges and Via Holes in ConductorBacked Coplanar Waveguides for MMIC Applications, " 1999 IEEE MTT-S Intl. Microwave Symp. Dig., pp. 709-712. 



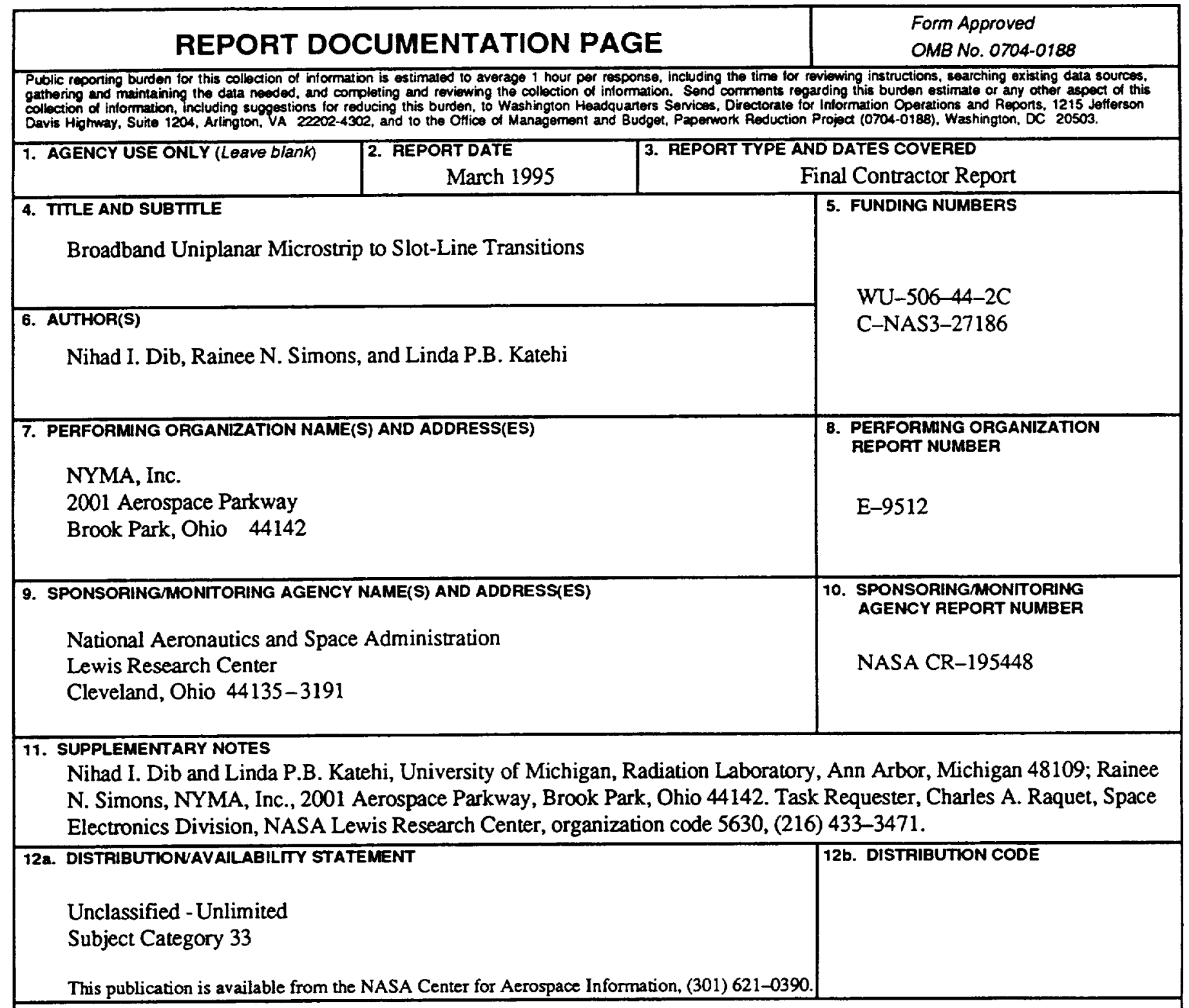

13. ABSTRACT (Maximum 200 words)

New in line uniplanar microstrip-to-slotline transitions for MIC/MMIC and phased array slotline antenna applications are described. Such transitions are compact and suitable to be used in an open environment or inside a package or a multichip module. The transitions share the concept of using a balun which consists of two microstrip lines connected to a slotline through a pair of coupled microstrips. In this paper, the transitions are studied theoretically using the FDTD technique and measured experimentally using an HP8510C Network Analyzer. For a back-to-back configuration, an insertion loss of less than $1.3 \mathrm{~dB}$ per transition is achieved over a $40 \% 3-\mathrm{dB}$ bandwidth with a minimum of $0.6 \mathrm{~dB}$ at the design frequency.

\begin{tabular}{|c|c|c|c|}
\hline \multirow{2}{*}{\multicolumn{3}{|c|}{$\begin{array}{l}\text { 14. SUBJECT TERMS } \\
\text { Microstrip; Slot-line; FDTD (Finite Difference Time Domain); Linearly tapered } \\
\text { slot antenna }\end{array}$}} & \multirow{2}{*}{\begin{tabular}{|c|} 
15. NUMBER OF PAGES \\
6 \\
$\begin{array}{c}\text { 16. PRICE CODE } \\
\text { AO2 } \\
\end{array}$ \\
UIMTATTON OF ABSTRACT
\end{tabular}} \\
\hline & & & \\
\hline $\begin{array}{l}\text { 17. SECURTYY CLASSIFICATION } \\
\text { OF REPORT }\end{array}$ & $\begin{array}{l}\text { 18. SECURTYY CLASSIFICATION } \\
\text { OF THIS PAGE }\end{array}$ & $\begin{array}{l}\text { 19. SECURITY CLASSIFICATION } \\
\text { OF ABSTRACT }\end{array}$ & 20. LIMITATION OF ABSTRACT \\
\hline Unclassified & Unclassified & Unclassified & \\
\hline
\end{tabular}

